\title{
BDNF: An Oncogene or Tumor Suppressor?
}

\author{
DANIEL P. RADIN ${ }^{1}$ and PARTH PATEL ${ }^{2}$ \\ ${ }^{1}$ Department of Pharmacology, Stony Brook University School of Medicine, Stony Brook, NY, U.S.A.; \\ ${ }^{2}$ College of Arts and Sciences, New York University, New York City, NY, U.S.A.
}

\begin{abstract}
Neurotrophins are a family of growth factors that are vital to the proper development of the central nervous system. Their effects on cells are governed by the expression and activation of the tyrosine kinase receptors TrkA, TrkB and TrkC. TrkB has been immensely implicated in mediating neuronal migration, development and differentiation. It has also been shown to protect several neuronal cell types from an array of cytotoxic stressors after activation by its conjugate ligand brainderived neurotrophic factor (BDNF). Over the past two decades, it has been shown that TrkB and BDNF are up-regulated in many types of cancers, conferring aggressive phenotypes underpinned by their resistance to several standard chemotherapeutic agents. This resistance to chemotherapy is modulated by the downstream targets of the TrkB receptor which include the well-characterized PI3K /Akt growth pathway, a hallmark of uncontrolled cancer cell growth and proliferation. Pre-clinical efforts to develop inhibitors of this receptor are promising, and such inhibitors also seem to sensitize cancer cells to standard chemotherapies. However, new evidence suggests that BDNF overexpression in the hypothalamus has immunoaugmenting properties, eliciting an increased anti-tumor immune response and reducing the activity of several proteins that would normally confer resistance to chemotherapeutic agents. In the current work, we provide a global analysis of the physiological consequences of TrkB receptor activation in vitro and discuss the dynamic consequences of TrkB activation in vivo. Finally, we propose a clinically-feasible option for increasing BDNF expression in the hypothalamus to more readily utilize the oncolytic effects of BDNF.
\end{abstract}

This article is freely accessible online.

Correspondence to: Daniel P. Radin, Department of Pharmacology, Stony Brook University School of Medicine, Stony Brook, 11790 NY, U.S.A. Tel: +1 2016790671, e-mail: danradin1@gmail.com

Key Words: BDNF, chemotherapeutic resistance, EGFR, PI3K, RhoA, Trkb, review.
Brain-derived neurotrophic factor (BDNF) has been widely implicated in the development, migration, differentiation and survival of fetal neurons (1). BDNF binds its conjugate receptor, TrkB, and initiates a signaling cascade marked by increased activity of the PI3K/Akt growth pathway leading to production of pro-migratory, antiapoptotic and pro-survival proteins $(2,3)$. It has been uniquely demonstrated that activation of the BDNF signaling pathway transactivates the epidermal growth factor receptor (EGFR) even in the absence of the endogenous EGF ligand (1). As a consequence, this augments the activity of phospholipase-C-y (PLCy) and of downstream targets such as various protein kinase $\mathrm{C}$ isoforms. BDNF/TrkB overexpression (Table I) confers a migratory phenotype to tumor cells and primes cancer cells to resist substantial genotoxic stressors, most of which are front-line chemotherapies (4-6). BDNF-mediated activation of TrkB also results in RAS activation (7), in turn accelerating cell-cycle progression and presumably contributing to poor prognosis of patients with cancers dependent on the BDNF signaling pathway $(8,9)$. BDNF and Trkb overexpression has also been shown to contribute to oncogenesis of aggressive neuroblastoma cells (10) and to post-chemotherapeutic recurrence of highly aggressive breast cancer stem cells (11), demonstrating that this signaling pathway seems to be ubiquitous in the progression of multiple cancers and dynamically regulates several aspects of tumor cell physiology.

Until recently, BDNF-mediated activation of the TrkB receptor was universally considered to have oncogenic consequences (12). While the signal transduction pathways initiated by TrkB receptor dimerization and activation are well elucidated, cancer cells express multiple isoforms of TrkB, which along with the tissue origin of the cancer ultimately govern the effect BDNF has on various cellular activities (1315). In fact, the first truncated version of the TrkB receptor, which we refer to as TrkB-T1, has been studied and reported to produce disparate and tissue-dependent effects on cellular signaling (see subsequent section). 
Table I. Expression of BDNF and TrkB in cancerous and normal tissues (Derived from Human Protein Atlas)*.

\begin{tabular}{|c|c|c|c|c|c|}
\hline \multirow[t]{2}{*}{ Cancer Type } & \multirow[t]{2}{*}{ Cell Type } & \multicolumn{2}{|c|}{$\mathrm{BDNF} * * *$} & \multicolumn{2}{|c|}{ TrkBNS } \\
\hline & & Cancerous Tissue & Normal Tissue & Cancerous Tissue & Normal Tissue \\
\hline Breast & Glandular & Moderate & ND & Moderate & Strong \\
\hline Carcinoid & Exocrine glandular & Moderate & ND & Strong & Moderate \\
\hline Cervical & Glandular & Moderate & ND & Moderate/Weak & Weak \\
\hline Colorectal & Glandular & Moderate & ND & Moderate & Moderate \\
\hline Endometrial & Glandular & Moderate & ND & Moderate & Moderate \\
\hline Glioma & Glial & Moderate/Weak & Weak & Strong/Moderate & Strong \\
\hline Head and neck & Glandular & Weak/ND & ND & Moderate & Moderate \\
\hline Liver & Bile duct & Moderate & ND & Moderate & Weak \\
\hline Lung & Respiratory Epithelial & Moderate/Weak & ND & Moderate/Weak & Moderate \\
\hline Lymphoma & Non-germinal center & Weak/ND & ND & Moderate/Weak & Moderate \\
\hline Melanoma & Melanocytes & Moderate & ND & Moderate/Weak & Moderate \\
\hline Ovarian & Epithelial & Moderate/Weak & NT & Moderate & NT \\
\hline Pancreatic & Exocrine glandular & Moderate & ND & Moderate & Moderate \\
\hline Prostate & Glandular & Moderate/Weak & ND & Moderate & Weak \\
\hline Renal & Cells in tubules & Moderate & ND & Moderate & Moderate \\
\hline Skin & Keratinocytes & Weak & ND & Weak & Moderate \\
\hline Stomach & Glandular & Moderate/Weak & ND & Moderate & Moderate \\
\hline Testis & Seminiferous Ducts & Strong/Moderate & ND & Moderate & Moderate \\
\hline Thyroid & Glandular & Weak & ND & Strong & Strong \\
\hline Urothelial & Urothelial & Moderate/Weak & ND & Moderate & Moderate \\
\hline
\end{tabular}

*Determined using immunohistochemical staining. ND: Not detected; NT; not tested. ${ }^{* * *} p=0.0001$, Wilcoxon signed-rank test, demonstrating significant overexpression in cancer biopsies. ${ }^{\mathrm{NS}} p=0.98$, Wilcoxon signed-rank test, demonstrating similar expression among normal tissue and cancer biopsies.

Recent studies have also suggested that although BDNF administration in vitro is globally oncogenic, BDNF administration in vivo may support an anti-tumor immune response. Mice living in enriched environments produce more BDNF in the hypothalamus and have significantly less circulating leptin plasma concentrations which may augment the cytotoxicity of multiple cell types of the immune system to induce tumor regression in multiple cancers $(15,16)$. Moreover, selective BDNF up-regulation in the hypothalamus mimics the effects of enriched environmental housing $(15,16)$. Due to the mounting evidence challenging the canonical oncogenic role of the BDNF/TrkB pathway, the present work seeks to provide a global analysis of in vitro trends and dynamic in vivo observations regarding the physiology of the BDNF/TrkB system. We also discuss the utility of BDNF/TrkB expression as prognostic markers and pharmacological means of up-regulating BDNF production in key brain regions to increase anti-tumor immune efficacy.

\section{BDNF Mediates BDNF/TrkB Synthesis}

Identifying mechanisms of BDNF synthesis and release into the extracellular matrix could prove valuable in slowing the growth of cancers relying on this signaling pathway. Earlier studies performed in primary neuronal cultures indicated that BDNF administration increased the production of BDNF mRNA and protein levels in a time- and dose-dependent fashion $(17,18)$. This positive feedback loop was mediated by BDNF-dependent activation of the PI3K/Akt pathway, which led to an increase in AMPA-type glutamate receptor levels. In turn, an increase in transmembrane AMPA receptor levels resulted in augmented BDNF mRNA expression and translation. Accordingly, pharmacological abrogation of the PI3K/Akt pathway or AMPA receptor blockade eliminated the BDNF-mediated increase in BDNF mRNA and protein levels (17).

More recent studies have indicated that TrkB receptor activation elicits phosphorylation of tyrosine 705 of STAT3, which transduces to amplify c-Myc and hypoxiainducible Factor 1-alpha (HIF1a) mRNA levels (19). Most notably, when the non-small cell lung cancer cell line A549 was serum-deprived for $24 \mathrm{~h}$, STAT3 activity declined initially but rebounded after $24 \mathrm{~h}$ of serum depletion, whereas pharmacological inhibition of TrkB by K252a, a well-characterized Trk inhibitor, continued to repress STAT3 activity. STAT3 and TrKB inhibition resulted in diminished Akt activity, revealing STAT3 as a potentially important signaling intermediate between TrkB and Akt (Figure 1). 


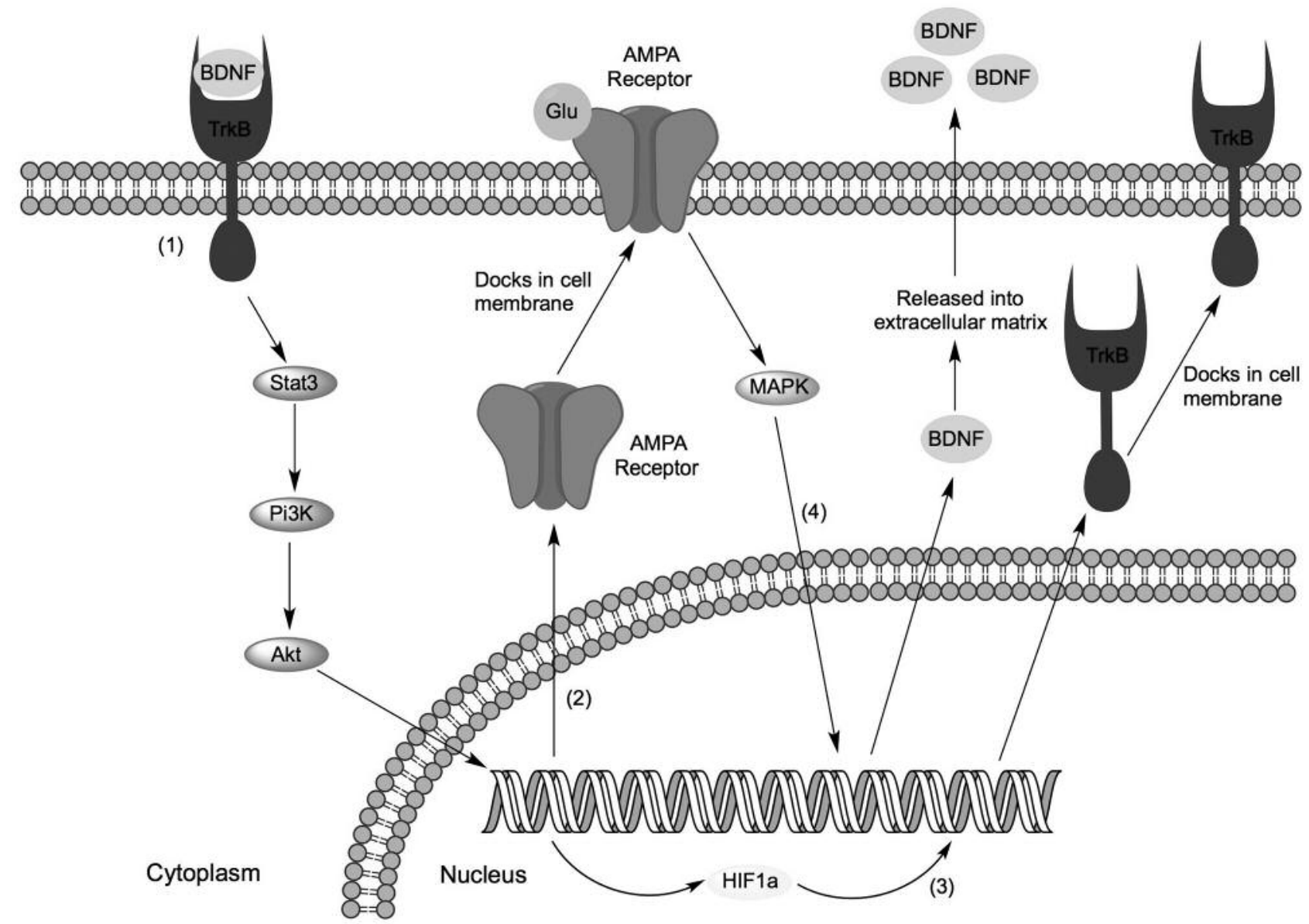

Figure 1. BDNF mediates its own synthesis in a positive feedback manner by activating TrkB (1) and primarily signaling for increased production of AMPA-type glutamate receptors (2). AMPA-glutamate receptor activation signals for increased BDNF expression (4). Accordingly, abrogating AMPA receptor signaling perturbs augmented BDNF production. BDNF also enhances HIFla levels which in turn increases TrkB expression (3).

It was separately demonstrated that HIF1a is a transcriptional activator of TrkB expression. In particular, neuroblastoma cells incubated in hypoxia $\left(1 \% \mathrm{O}_{2}\right)$ for $24 \mathrm{~h}$ up-regulated TrkB expression 30-fold whereas expression of other neurotrophin receptors did not significantly differ (20). Neuroblastoma cell incubation in normoxia $\left(21 \% \mathrm{O}_{2}\right)$ with the hypoxia mimic 2,2'-dipyridil also enhanced TrkB expression $\sim 8$-fold. This effect was absent in cells lacking HIF1a or diminished in cells expressing an siRNA targeting HIF1a, confirming this protein's involvement in the regulation of TrkB expression (20) (Figure 1). It is also pertinent to note that cells incubated in hypoxia displayed higher migratory activity, indicating that up-regulation of TrkB can facilitate migration of cancerous cells (M20). Nicotinic receptor activation has also been shown to promote BDNF release by neuroblastoma cells, providing a causal link between cigarette smoke and cancer facilitation (21).

\section{BDNF role in Migration and Oncogenesis}

TrkB/EGFR crosstalk: Partners in crime. The immediate consequences of TrkB activation are vital to understanding the physiology of this pathway. TrkB has been shown to cross-talk with EGFR signaling pathway, a growth factor receptor commonly up-regulated in many cancers. In noncancerous tissue (1) and in cancerous tissue (22), BDNF administration results in the expected increase in TrkB phosphorylation and also results in EGFR phosphorylation and activation independent of EGF concentration in serum media. EGF-mediated transactivation of TrkB is not only vital for the migration of embryonic cortical neurons (1), but also strongly facilitates migration of lung cancer cells (22) and proliferation and migration of ovarian cancer cells (24). Furthermore, BDNF administration abrogates the oncolytic effects of EGFR inhibition, making it more difficult to 
silence constitutively active growth pathways, often considered hallmarks of cancer. Fortunately, it has been demonstrated that dual inhibition of EGFR and TrkB synergistically suppresses colon cancer cell proliferation (23). TrkB inhibition has also been shown to mitigate the pro-migratory effects of EGF administration and EGFR inhibition has also been shown to mitigate the pro-migratory effects of BDNF administration in lung cancer cells (24). Taken together, the EGFR and BDNF signaling pathways seem to be intricately connected and are able to compensate for one another in the case of singular inhibition, but available evidence also suggests that their intricate connection yields their signaling pathways susceptible to indirect inhibition, which can aid in targeting tumors where either pathway is constitutively active.

Role in epithelial to mesenchymal transition and anoikis. Tumors become increasingly difficult to treat when they metastasize to other organs in the body. This is indicative of high-grade cancers which are usually associated with terminal diagnoses (31). As such, much research has been done to determine the molecular mechanisms by which portions of solid tumors metastasize from the original cancerous mass and migrate to other organs in the body where nutrients are plentiful such as the lungs or the brain. When metastasizing, tumor cells may undergo anchorageindependent cell death or anoikis (18). The other issue is that tumor cells must transition from an epithelial nature to a mesenchymal one (EMT) by modulating gene expression, where they are more equipped to survive short travel through the blood to distant organs $(18,25)$.

Due to how short-lived some of these alterations in gene expression can be, the study of anoikis and EMT has been slow until recently. What is of considerable interest is that the $\mathrm{BDNF} / \mathrm{TrkB}$ pathway has been shown to mediate the resistance to anoikis of multiple cancers $(9,18,25-29)$. It was shown that in multiple cancers, metastatic tumor cells have up-regulated TrkB and BDNF compared to tumor cells that did not metastasize $(18,26,29)$. Furthermore, tumor cells were resistant to anchorage-independent cell death, or anoikis, because TrkB activation resulted in constitutive PI3K activity and survival for the duration of the migration to neighboring tissues (18), supporting the pro-survival role of BDNF as tumor cells migrate. This data may explain previous reports citing the general pro-migratory role of $\operatorname{BDNF}(3,29-32)$.

Role in tumor formation and relapse. BDNF has also been implicated in mediating oncogenic transformation $(10,33)$ and tumor relapse (11) after supposed successful treatment of cancer. In a neural crest-derived cell line forced to overexpress TrkB, such cells formed rapidly growing tumors in mice 10 days after implantation and killed all mice within 7 days of tumor formation (10). Brain tumor-initiating cells, were also shown to be able to survive without canonical growth factors as long as they overexpressed TrkB and produced BDNF, supposedly releasing it in an autocrine fashion to sustain their own survival (33). Tumor-initiating cells are often referred to as cancer stem cells, due to the fact that they are cancerous in nature but also express stem cell markers and are slowly dividing. Therefore, front-line chemotherapies that target rapidly dividing tumor cells are unable to kill off these cells (11). It is believed that these cells are the main contributors to tumor relapse after what is considered to be successful cancer treatment (11). At the same time, TrkB-positive cancer stem cells were shown to play a massive role in tumor relapse after treatment of mice harboring triple-negative breast cancers (11). These data demonstrate that treatment with a TrkB inhibitor even after treatment with chemotherapy may prolong disease-free survival of cancer patients. TrkB inhibitors may also play a large clinical role preventing tumor reformation or tumor formation in other tissue types of patients who have been shown to be at high risk for certain types of cancer.

\section{BDNF: A Governor of Chemotherapeutic Sensitivity}

The first report indicating that BDNF may modulate a cancer cell's sensitivity to chemotherapy was published two decades ago. Researchers determined that vinblastine was significantly less efficacious in neuroblastoma cells expressing a TrkB plasmid. In these transfected cells vinblastine was less able to deter tubulin polymerization and intracellular vinblastine levels were lower in cells expressing the TrkB plasmid, though multi-drug resistance pump (MDR) expression, protein levels or activity were not altered by introduction of the TrkB plasmid (34). Since then, reports have identified the $\mathrm{BDNF} /$ TrkB signaling pathway as a universal attenuator of chemotherapeutic efficacy. Jaboin et al. reported that BDNF rescued neuroblastoma cells from cisplatin, etoposide and vinblastine in a dose-dependent fashion whereas other neurotrophins failed to rescue neuroblastoma cells $(4,35)$. PI3K but not MAPK inhibition abrogated BDNF's ability to protect cancer cells from etoposide $(4,35)$. Chemotherapeutic resistance conferred to head and neck squamous cell carcinoma by BDNF administration was shown to be mediated by an up-regulation of MDR1 (5), contradicting the results obtained by Scala et al. (34). This data shows that while BDNF is universally cytoprotective in vitro, the mode by which BDNF protects cancer cells from various chemotherapies is probably dependent on the cell type.

BDNF-mediated protection from chemotherapy was also mediated by the down-regulation of Bim, a pro-apoptotic protein that facilitates mitochondrial-mediated or intrinsic apoptosis $(5,36)$. Furthermore, it is possible that BDNF promotes resistance to extrinsic apoptosis by up-regulating production of proteins associated with halting the activation 
of transmembrane death receptors. For example, cisplatin operates in triple-negative breast cancer cells by inducing Fas-mediated Caspase-8-dependent apoptosis after induction of DNA damage, but this effect is mitigated by Fas apoptotic inhibitory molecule 2 (FAIM2) (37). Production of this protein is directly correlated to PI3K activity, which is downstream of TrkB. FAIM2 may serve as a viable candidate for pinpointing a specific mechanism by which BDNF halts cisplatin efficacy, though further studies are needed to confirm this possibility.

It became evident that while BDNF antagonizes chemotherapeutic efficacy, cancer cells that dependent upon the BDNF/TrkB signaling system for sustained proliferation and invasion would most likely be more susceptible to chemotherapeutic treatment after genetic or pharmacological inhibition of the BDNF pathway. Mice inoculated with breast cancer cells and treated with antibodies against BDNF display slower tumor progression compared to mice treated with control antibodies (38). These results were recapitulated in a mouse model of uterine sarcoma (39) and neuroblastoma $(40,41)$. Mice flanked with choriocarcinoma xenografts and subsequently treated with K252a exhibited drastically slower tumor growth paralleled with a tumor-specific 15-fold increase in caspase-3/7 levels (42).

Cisplatin was also shown to increase production of microRNA-16 (miR-16) in neuroblastoma cells, which in turn reduced the levels of BDNF in a concentration-dependent manner (43). Inhibition of BDNF production resulted in stunted neuroblastoma tumor growth in vivo (43) suggesting that, at least in part, BDNF's antagonism of cisplatin efficacy is due to the compensation of decreased BDNF levels mediated by cisplatin's activation of miR-16. Other miRs that act antagonistic to BDNF production include MiR-206 in gastric cancer (44) and MiR-107 in non-small cell lung cancer (2). Most notably, BDNF pathway inhibition sensitized Ewing sarcoma cells to a myriad of chemotherapies (6). Pharmacological inhibition of Trk by the use of K252a strongly potentiated the effects of vincristine, etoposide and doxorubicin (6). This recent report lends strong credibility to the notion that Trk inhibition would have massive utility in the clinic, either as a sole treatment regimen or as a neoadjuvant treatment to sensitize cancers to the oncolytic effects of standard chemotherapies on the market.

\section{BDNF's Disparate Effects on RhoA GTPase}

Though BDNF largely exerts an oncogenic effect and induces massive chemotherapeutic resistance in many cancers, the specific effects are dependent on the cellular context. For example, reports have indicated that BDNF positively regulates migratory behavior in non-small cell lung cancer (2), gastric cancer (44) and nasopharyngeal carcinoma. However, direct BDNF injection into murine glioma xenografts results in decreased propensity toward migration (15), suggesting some heterogeneity in the cellular response to BDNF. A potential insight to this quandary can be uncovered by examining the specific isoform of TrkB predominantly expressed from cancer to cancer. In hepatocellular carcinoma, activation of the full length TrkB by BDNF initiates a signaling cascade that results in increased activity of RhoA, a small $\mathrm{G}$ protein belonging to the Ras superfamily (45). This $\mathrm{G}$ protein dynamically regulates actin polymerization which in turn governs cellular locomotion. The truncated form of TrkB, Trkb-T1, physically dissociates from an endogenous inhibitor of RhoA following BDNF administration, known as Rho guanine nucleotide dissociation inhibitor (Rho GDI) (13, 15), which suppresses metastasis. Conversely, activation of Trkb-T1 in pancreatic adenocarcinoma has been shown to sequester Rho GDI and induce pancreatic cancer metastasis (46), suggesting that more work is required to be able to precisely predict what effect BDNF administration will have on RhoA and subsequently the migratory phenotype of the cancer of interest.

\section{Immunoaugmentation: Awakening the Sleeping Giant}

It is of critical importance for anti-cancer agents entering the clinic to be vigorously studied on not only the effect it has on the cancer of interest, but on other bodily systems such as the immune system, neuronal cell types and other rapidly dividing cells of non-malignant origin. Oftentimes, anticancer agents target at least one of these types of tissues, underpinning their propensity for exhibiting large side effects. With specific emphasis on the immune system, oftentimes immunodeficient mice are needed to test the efficacy of candidate drugs on human cancer xenografts. While such pre-clinical models tend to hold up in the clinic, the use of this model ignores the potential effects oncolytic drugs may have on the immune system.

In a pioneering study by Cao et al. (16), researchers found that housing mice receiving melanoma xenografts in enriched environmental (EE) housing starkly halted tumor growth when compared to that of mice living in standard environments (SE). Tumors of EE mice exhibited reduced Aakt activity and reduced expression of several pro-survival proteins such as VEGF, IGF-1 (47) and p-ERK (16). Natural killer and T-cell cytotoxicity was significantly augmented in EE mice, which researchers believed governed the oncolytic effect of EE housing. BDNF expression increased in the hypothalamus of EE mice over the course of the monitoring period. Hypothalamic knockdown of BDNF abrogated the EE-induced tumor resistance (16), highlighting BDNF as a major player in mediating the oncolytic effects of $\mathrm{EE}$ housing. These data were comparable to that obtained in mice transplanted with breast cancer cells (47 2014). Most 
intriguing was that in both studies, hypothalamic BDNF upregulation did not exert an anti-tumor phenotype if the mice were overweight $(16,47)$, suggesting that BDNF upregulation in the hypothalamus is not solely sufficient to ensure tumor regression in mice.

Follow-up studies indicated that environmental and genetic activation of hypothalamic BDNF augments T-cell cytotoxicity to confer an anti-cancer phenotype in melanoma (48) and in glioma (15). This suggests that while autocrine BDNF signaling will most likely promote cancer progression and aggressiveness, BDNF up-regulation restricted to the central nervous system more readily produces a shift toward oncolysis rather than oncogenesis, even if the tumor is localized to the central nervous system (15). The immunoaugmenting capacity of BDNF may be explained by the fact that BDNF is required for proper T-cell maturation (49). These data also indicate that the environment in which cancer patients find themselves during the course of treatment may significantly influence clinical outcomes.

\section{Moving Forward}

The available literature virtually demonstrates in all cases that BDNF accelerates cancer progression in many areas of its physiology and that this effect is observed in many cancers. Thus, it would be impractical to continue to study the effects of BDNF on the full TrkB isoform because of how well the cellular consequences have already been characterized. It would be fruitful to continue to investigate the in vivo effects of BDNF since recent literature has demonstrated the various oncolytic effects of BDNF administration observed in vivo. Furthermore, efforts should be directed toward increasing BDNF production in the hypothalamus since pre-clinical reports suggest that augmenting BDNF production in this brain region can induce massive tumor regression in multiple types of cancer.

The search for a drug that can increase BDNF production in the CNS began long before the connection was made between BDNF and cancer. BDNF possesses many corrective properties in multiple neurodegenerative and neuropsychiatric disorders (50-52). Cortex Pharmaceuticals, Lilly Pharmaceuticals and Servier Pharmaceuticals have all spent decades trying to establish drugs that could safely augment BDNF levels in the brain in an effort to mitigate the effects of neurodegenerative diseases. Most of these efforts have been put into developing positive allosteric modulators of the AMPA receptor, as they have shown to increase BDNF production in several brain regions up to 20 -fold within a few days of acute administration. While such encouraging early pre-clinical results seem promising, efforts to get these drugs into the clinic have been halted by the unintended seizurogenic effects at doses related to what would be needed to achieve BDNF augmentation. Serendipitously, RespireRx (previously Cortex) Pharmaceuticals recently reported on one of the first AMPA receptor positive allosteric modulator (termed Ampakines) that corrects age-related deficits in memory while not exhibiting the seizurogenic side effects of predecessor compounds (53). The predecessor compounds of this drug, CX1846, have all been shown to elevate BDNF levels at doses CX1846 corrects age-related memory issues without exerting unwanted side effects. It would be of considerable clinical interest to investigate Ampakines as a means to up-regulate BDNF in the hypothalamus by positively modulating hypothalamic AMPA receptors (54) since targeted genetic therapy is at its infancy.

\section{Concluding Remarks}

In the past two decades, considerable strides have been made in understanding the relevance of BDNF physiology in multiple areas of cancer progression. TrkB antagonists in clinical development should certainly reduce cancer burden for patients, augment the oncolytic effects of widely used cancer drugs, prevent new cancers from forming and hopefully prevent cancer relapse by eliminating TrkBpositive cancer stem cells. Research should continue to examine the disparate effects of TrkB isoforms and especially examine what signaling pathways predominate when tumors express multiple isoforms. Finally, researchers should begin to examine clinically relevant means of upregulating $\mathrm{BDNF}$ in the hypothalamus to induce an antitumor immune response which may also increase the efficacy of common anti-cancer drugs.

\section{Conflicts of Interest}

The Authors declare no conflict of interest pertaining to the current work.

\section{References}

1 Puehringer $\mathrm{D}$, Orel $\mathrm{N}$, Luningschror $\mathrm{P}$, Subramanian $\mathrm{N}$, Herrmann T, Chao MV and Sendtner M: Egf transactivation of trk receptors regulates the migration of newborn cortical neurons. Nat Neurosci 16(4): 407-415, 2013.

2 Xia H, Li Y and Lv X: Microrna-107 inhibits tumor growth and metastasis by targeting the bdnf-mediated pi3k/akt pathway in human non-small lung cancer. Int J Oncol 49(4): 1325-1333, 2016.

3 Dewitt J, Ochoa V, Urschitz J, Elston M, Moisyadi S and Nishi $\mathrm{R}$ : Constitutively active trkb confers an aggressive transformed phenotype to a neural crest-derived cell line. Oncogene 33(8): 977-985, 2014.

4 Heinen TE, Dos Santos RP, da Rocha A, Dos Santos MP, Lopez PL, Silva Filho MA, Souza BK, Rivero LF, Becker RG, Gregianin LJ, Brunetto AL, Brunetto AT, de Farias CB and Roesler R: Trk inhibition reduces cell proliferation and potentiates the effects of chemotherapeutic agents in ewing sarcoma. Oncotarget 7(23): 34860-34880, 2016. 
5 Jaboin J, Kim CJ, Kaplan DR and Thiele CJ: Brain-derived neurotrophic factor activation of trkb protects neuroblastoma cells from chemotherapy-induced apoptosis via phosphatidylinositol 3'-kinase pathway. Cancer Res 62(22): 6756-6763, 2002.

6 Jia S, Wang W, Hu Z, Shan C, Wang L, Wu B, Yang Z, Yang X and Lei D: Bdnf mediated trkb activation contributes to the emt progression and the poor prognosis in human salivary adenoid cystic carcinoma. Oral Oncol 51(1): 64-70, 2015.

7 Lee J, Jiffar T and Kupferman ME: A novel role for bdnf-trkb in the regulation of chemotherapy resistance in head and neck squamous cell carcinoma. PLoS One 7(1): e30246, 2012.

8 Okamura K, Harada T, Wang S, Ijichi K, Furuyama K, Koga T, Okamoto T, Takayama K, Yano T and Nakanishi Y: Expression of trkb and bdnf is associated with poor prognosis in non-small cell lung cancer. Lung Cancer 78(1): 100-106, 2012.

9 Sinkevicius KW, Kriegel C, Bellaria KJ, Lee J, Lau AN, Leeman KT, Zhou P, Beede AM, Fillmore CM, Caswell D, Barrios J, Wong KK, Sholl LM, Schlaeger TM, Bronson RT, Chirieac LR, Winslow MM, Haigis MC and Kim CF: Neurotrophin receptor trkb promotes lung adenocarcinoma metastasis. Proc Natl Acad Sci USA 111(28): 10299-10304, 2014.

10 Yin B, Ma ZY, Zhou ZW, Gao WC, Du ZG, Zhao ZH and Li QQ: The trkb+ cancer stem cells contribute to postchemotherapy recurrence of triple-negative breast cancers in an orthotopic mouse model. Oncogene 34(6): 761-770, 2015.

11 Zhang SY, Hui LP, Li CY, Gao J, Cui ZS and Qiu XS: More expression of bdnf associates with lung squamous cell carcinoma and is critical to the proliferation and invasion of lung cancer cells. BMC Cancer 16: 171, 2016.

12 Thiele CJ, Li Z and McKee AE: On trk--the trkb signal transduction pathway is an increasingly important target in cancer biology. Clin Cancer Res 15(19): 5962-5967, 2009.

13 Ohira K, Homma KJ, Hirai H, Nakamura S and Hayashi M: Trkb-t1 regulates the rhoa signaling and actin cytoskeleton in glioma cells. Biochem Biophys Res Commun 342(3): 867-874, 2006.

14 Harada T, Yatabe Y, Takeshita M, Koga T, Yano T, Wang Y and Giaccone G: Role and relevance of trkb mutations and expression in non-small cell lung cancer. Clin Cancer Res 17(9): 2638-2645, 2011

15 Garofalo S, D'Alessandro G, Chece G, Brau F, Maggi L, Rosa A, Porzia A, Mainiero F, Esposito V, Lauro C, Benigni G, Bernardini G, Santoni A and Limatola C: Enriched environment reduces glioma growth through immune and non-immune mechanisms in mice. Nat Commun 6: 6623, 2015.

16 Cao L, Liu X, Lin EJ, Wang C, Choi EY, Riban V, Lin B and During MJ: Environmental and genetic activation of a brainadipocyte bdnf/leptin axis causes cancer remission and inhibition. Cell 142(1): 52-64, 2010.

17 Xiong H, Futamura T, Jourdi H, Zhou H, Takei N, DiversePierluissi M, Plevy S and Nawa $\mathrm{H}$ : Neurotrophins induce bdnf expression through the glutamate receptor pathway in neocortical neurons. Neuropharmacology 42(7): 903-912, 2002 .

18 Bao W, Qiu H, Yang T, Luo X, Zhang H and Wan X: Upregulation of trkb promotes epithelial-mesenchymal transition and anoikis resistance in endometrial carcinoma. PLoS One 8(7): e70616, 2013

19 Chen B, Liang Y, He Z, An Y, Zhao W and Wu J: Autocrine activity of bdnf induced by the stat 3 signaling pathway causes prolonged trkb activation and promotes human non-small-cell lung cancer proliferation. Sci Rep 6: 30404, 2016.

20 Martens LK, Kirschner KM, Warnecke C and Scholz H: Hypoxia-inducible factor-1 (hif-1) is a transcriptional activator of the trkb neurotrophin receptor gene. J Biol Chem 282(19): 14379-14388, 2007.

21 Serres F and Carney SL: Nicotine regulates sh-sy5y neuroblastoma cell proliferation through the release of brainderived neurotrophic factor. Brain Res 1101(1): 36-42, 2006.

22 Gotz R and Sendtner M: Cooperation of tyrosine kinase receptor trkb and epidermal growth factor receptor signaling enhances migration and dispersal of lung tumor cells. PLoS One 9(6): e100944, 2014.

23 de Farias CB, Heinen TE, dos Santos RP, Abujamra AL, Schwartsmann G and Roesler R: Bdnf/trkb signaling protects ht29 human colon cancer cells from egfr inhibition. Biochem Biophys Res Commun 425(2): 328-332, 2012.

24 Qiu L, Zhou C, Sun Y, Di W, Scheffler E, Healey S, Kouttab N, Chu W and Wan Y: Crosstalk between egfr and trkb enhances ovarian cancer cell migration and proliferation. Int $\mathbf{J}$ Oncol 29(4): 1003-1011, 2006.

25 Kupferman ME, Jiffar T, El-Naggar A, Yilmaz T, Zhou G, Xie T, Feng L, Wang J, Holsinger FC, Yu D and Myers JN: Trkb induces emt and has a key role in invasion of head and neck squamous cell carcinoma. Oncogene 29(14): 2047-2059, 2010.

26 Ricci A, De Vitis C, Noto A, Fattore L, Mariotta S, Cherubini E, Roscilli G, Liguori G, Scognamiglio G, Rocco G, Botti G, Giarnieri E, Giovagnoli MR, De Toma G, Ciliberto G and Mancini R: Trkb is responsible for emt transition in malignant pleural effusions derived cultures from adenocarcinoma of the lung. Cell Cycle 12(11): 1696-1703, 2013.

27 Alonso-Alconada L, Eritja N, Muinelo-Romay L, Barbazan J, Lopez-Lopez R, Matias-Guiu X, Gil-Moreno A, Dolcet X and Abal M: Etv5 transcription program links bdnf and promotion of emt at invasive front of endometrial carcinomas. Carcinogenesis 35(12): 2679-2686, 2014.

28 Dudas J, Bitsche M, Schartinger V, Falkeis C, Sprinzl GM and Riechelmann H: Fibroblasts produce brain-derived neurotrophic factor and induce mesenchymal transition of oral tumor cells. Oral Oncol 47(2): 98-103, 2011.

29 Smit MA, Geiger TR, Song JY, Gitelman I and Peeper DS: A twist-snail axis critical for trkb-induced epithelial-mesenchymal transition-like transformation, anoikis resistance, and metastasis. Mol Cell Biol 29(13): 3722-3737, 2009.

30 Huang SM, Lin C, Lin HY, Chiu CM, Fang CW, Liao KF, Chen DR and Yeh WL: Brain-derived neurotrophic factor regulates cell motility in human colon cancer. Endocr Relat Cancer 22(3): 455-464, 2015.

31 Kim MS, Lee WS and Jin W: Trkb promotes breast cancer metastasis via suppression of runx3 and keap1 expression. Mol Cells 39(3): 258-265, 2016.

32 Lin CY, Chen HJ, Li TM, Fong YC, Liu SC, Chen PC and Tang $\mathrm{CH}$ : Beta5 integrin up-regulation in brain-derived neurotrophic factor promotes cell motility in human chondrosarcoma. PLoS One 8(7): e67990, 2013.

33 Lawn S, Krishna N, Pisklakova A, Qu X, Fenstermacher DA, Fournier M, Vrionis FD, Tran N, Chan JA, Kenchappa RS and Forsyth PA: Neurotrophin signaling via trkb and trkc receptors promotes the growth of brain tumor-initiating cells. J Biol Chem 290(6): 3814-3824, 2015 
34 Scala S, Wosikowski K, Giannakakou P, Valle P, Biedler JL, Spengler BA, Lucarelli E, Bates SE and Thiele CJ: Brainderived neurotrophic factor protects neuroblastoma cells from vinblastine toxicity. Cancer Res 56(16): 3737-3742, 1996.

35 Jaboin J, Hong A, Kim CJ and Thiele CJ: Cisplatin-induced cytotoxicity is blocked by brain-derived neurotrophic factor activation of trkb signal transduction path in neuroblastoma. Cancer Lett 193(1): 109-114, 2003.

$36 \mathrm{Li} \mathrm{Z,} \mathrm{Zhang} \mathrm{J,} \mathrm{Liu} \mathrm{Z,} \mathrm{Woo} \mathrm{CW} \mathrm{and} \mathrm{Thiele} \mathrm{CJ:} \mathrm{Down-regulation}$ of bim by brain-derived neurotrophic factor activation of trkb protects neuroblastoma cells from paclitaxel but not etoposide or cisplatin-induced cell death. Cell Death Differ 14(2): 318-326, 2007.

37 Radin D, Lippa A, Patel P and Leonardi D: Lifeguard inhibition of fas-mediated apoptosis: A possible mechanism for explaining the cisplatin resistance of triple-negative breast cancer cells. Biomed Pharmacother 77: 161-166, 2016.

38 Vanhecke E, Adriaenssens E, Verbeke S, Meignan S, Germain E, Berteaux N, Nurcombe V, Le Bourhis X and Hondermarck H: Brain-derived neurotrophic factor and neurotrophin-4/5 are expressed in breast cancer and can be targeted to inhibit tumor cell survival. Clin Cancer Res 17(7): 1741-1752, 2011.

39 Makino K, Kawamura K, Sato W, Kawamura N, Fujimoto T and Terada Y: Inhibition of uterine sarcoma cell growth through suppression of endogenous tyrosine kinase b signaling. PLoS One 7(7): e41049, 2012.

40 Iyer R, Evans AE, Qi X, Ho R, Minturn JE, Zhao H, Balamuth $\mathrm{N}$, Maris JM and Brodeur GM: Lestaurtinib enhances the antitumor efficacy of chemotherapy in murine xenograft models of neuroblastoma. Clin Cancer Res 16(5): 1478-1485, 2010.

41 Iyer R, Wehrmann L, Golden RL, Naraparaju K, Croucher JL, MacFarland SP, Guan P, Kolla V, Wei G, Cam N, Li G, Hornby $\mathrm{Z}$ and Brodeur GM: Entrectinib is a potent inhibitor of trk-driven neuroblastomas in a xenograft mouse model. Cancer Lett 372(2): 179-186, 2016.

42 Kawamura N, Kawamura K, Manabe M and Tanaka T: Inhibition of brain-derived neurotrophic factor/tyrosine kinase b signaling suppresses choriocarcinoma cell growth. Endocrinology 151(7): 3006-3014, 2010.

43 Sun YX, Yang J, Wang PY, Li YJ, Xie SY and Sun RP: Cisplatin regulates sh-sy5y cell growth through down-regulation of bdnf via mir-16. Oncol Rep 30(5): 2343-2349, 2013.

44 Ren J, Huang HJ, Gong Y, Yue S, Tang LM and Cheng SY: Microrna-206 suppresses gastric cancer cell growth and metastasis. Cell Biosci 4: 26, 2014.

45 Guo D, Sun W, Zhu L, Zhang H, Hou X, Liang J, Jiang X and Liu C: Knockdown of bdnf suppressed invasion of hepg2 and hcclm3 cells, a mechanism associated with inactivation of rhoa or rac1 and actin skeleton disorganization. APMIS 120(6): 469476, 2012
46 Li Z, Chang Z, Chiao LJ, Kang Y, Xia Q, Zhu C, Fleming JB, Evans DB and Chiao PJ: Trkbt1 induces liver metastasis of pancreatic cancer cells by sequestering rho gdp dissociation inhibitor and promoting rhoa activation. Cancer Res 69(19): 7851-7859, 2009.

47 Liu X, McMurphy T, Xiao R, Slater A, Huang W and Cao L: Hypothalamic gene transfer of bdnf inhibits breast cancer progression and metastasis in middle age obese mice. Mol Ther 22(7): 1275-1284, 2014.

48 Xiao R, Bergin SM, Huang W, Slater AM, Liu X, Judd RT, Lin EJ, Widstrom KJ, Scoville SD, Yu J, Caligiuri MA and Cao L: Environmental and genetic activation of hypothalamic bdnf modulates t-cell immunity to exert an anticancer phenotype. Cancer Immunol Res 4(6): 488-497, 2016.

49 Linker RA, Lee DH, Flach AC, Litke T, van den Brandt J, Reichardt HM, Lingner T, Bommhardt U, Sendtner M, Gold R, Flugel A and Luhder F: Thymocyte-derived bdnf influences t-cell maturation at the $\mathrm{dn} 3 / \mathrm{dn} 4$ transition stage. Eur J Immunol 45(5): 1326-1338, 2015.

50 Ogier M, Wang H, Hong E, Wang Q, Greenberg ME and Katz DM: Brain-derived neurotrophic factor expression and respiratory function improve after ampakine treatment in a mouse model of rett syndrome. J Neurosci 27(40): 10912-10917, 2007.

51 Simmons DA, Rex CS, Palmer L, Pandyarajan V, Fedulov V, Gall $\mathrm{CM}$ and Lynch G: Up-regulating bdnf with an ampakine rescues synaptic plasticity and memory in huntington's disease knockin mice. Proc Natl Acad Sci USA 106(12): 4906-4911, 2009.

52 Clarkson AN, Overman JJ, Zhong S, Mueller R, Lynch G and Carmichael ST: Ampa receptor-induced local brain-derived neurotrophic factor signaling mediates motor recovery after stroke. J Neurosci 31(10): 3766-3775, 2011.

53 Radin DP, Zhong S, Purcell R and Lippa A: Acute ampakine treatment ameliorates age-related deficits in long-term potentiation. Biomed Pharmacother 84: 806-809, 2016.

54 Ferreira-Neto HC, Antunes VR and Stern JE: Atp stimulates rat hypothalamic sympathetic neurons by enhancing ampa receptormediated currents. J Neurophysiol 114(1): 159-169, 2015. 